\title{
Why do Groups Cooperate More than Individuals to Reduce Risks
}

\author{
Min Gong*, Jonathan Baron\#, Howard Kunreuther+
}

* Center for Research on Environmental Decisions, Columbia University, 406 Schermerhorn Hall - MC 5501, 1190 Amsterdam Ave, New York, NY 10027. Email address: mg3030@columbia.edu.

\# Department of Psychology, University of Pennsylvania. Email address: baron@psych.upenn.edu.

+ Cecilia Yen Koo Professor of Decisions Sciences and Public Policy at the Wharton School, University of Pennsylvania and co-director of the Wharton Risk Management and Decision Processes Center.E-mail address: Kunreuther@wharton.upenn.edu. 
Abstract: Previous research has discovered a curious phenomenon: groups cooperate less than individuals in a deterministic prisoner's dilemma game but cooperate more than individuals when uncertainty is introduced into the game. We conduct two studies to examine three possible processes that may have driven groups to be more cooperative than individuals to reduce risks: group risk concern, group cooperation expectation, and social pressure. We find that ex-post guilt aversion and ex-post blame avoidance under uncertainty cause group members to be more risk concerned than individuals. This concern drives groups to choose the cooperation (and risk-reduction) strategy more frequently than individuals do. Groups also have higher cooperation expectations for the other group than individuals have for the other individual. We find no evidence of social pressure pushing groups to be nicer and more cooperative than individuals.

Key words: group decision, uncertainty, cooperation, experimental economics

\section{JEL code: D81}

Acknowledgements: We appreciate helpful comments and suggestions from Jason Dana, David Hardisty, David Krantz, Sabine Max, Deborah Small, Ewa Szymanska, Elke Weber, and participants at the SJDM 2009 Annual Conference, Jonathan Baron lab meeting, and the CRED lab meeting. We thank the Wharton behavior lab and David Hynes for data collecting and compiling. This research is supported by the Russell Ackoff Doctoral Student Fellowship at the Wharton Risk Management and Decision Processes Center and by National Science Foundation grant CMS-0527598. 


\section{Introduction}

We recently reported a curious phenomenon in comparing group decisions with those of individuals in a game theoretic setting. In a deterministic prisoner's dilemma (DPD) game, 3-person groups cooperated less than did individuals (32\% vs. $78 \%$ ), but cooperated more than individuals in a stochastic Prisoner's Dilemma (SPD) game (52\% vs. 22\%) (Gong et al., 2009). In these two-agent controlled laboratory experiments, groups always played against another group, and an individual against another individual. Tables 1 and 2 illustrate the payoff matrices for the SPD game and DPD game respectively where a cooperative decision is one where the agent invests in protection. Negative numbers represent losses. Percentages are probabilities of various outcomes as a function of the specific decision by each of the agents. The expected values in the SPD game are the corresponding values in the DPD game. The Nash equilibrium, assuming risk neutrality and using maximization of expected values as a criterion for choosing between alternatives is (Not Invest, Not Invest).

[Insert Table 1 and 2 here]

In the SPD game, each agent (i.e. a three-person group or an individual) decides whether or not to pay an investment cost (45) to reduce its own risk of experiencing a larger loss (100). If one agent invests, both agents' likelihoods of suffering a loss are reduced, but the investor's risk is reduced more than her counterpart's (from 52\% to $20 \%$ vs. from $52 \%$ to $40 \%$ ). Joint cooperation (both investing) eliminates uncertainty completely and each player incurs only the cost of investment cost only.

The stochastic prisoner's dilemma (SPD) is a special case of an interdependent security game (Heal and Kunreuther, 2007), which characterizes a variety of problems in the real world, such as airline security (e.g., the Pan Am 103 crash), emission reduction by multiple nationals in the global warming problem, bankruptcy of an entire company caused by a catastrophic loss from one of its divisions, etc. In the latter connection a recent example is the potential collapse of the American International Group (A.I.G.), the world's largest insurer, during the financial crisis of 2008, which was the result of a 377person London Unit known as A.I.G. Financial Products that was run with almost complete autonomy from the parent company (Kunreuther 2009).

There were two interesting group-individual differences in Gong et al. (2009). First, groups cooperated less than individuals in the DPD game. This behavior is consistent with a well replicated phenomenon in social psychology that groups are usually more competitive and less cooperative than individuals in the context of mixedmotive matrix games, such as the prisoner's dilemma. In social psychology, this groupbeing-more-competitive-than-individual phenomenon is termed the "intergroup interindividual discontinuity effect" (for a review of studies on this behavior see Wildschut et al., 2007).

Second, when uncertainty is introduced into the SPD game, the discontinuity effect not only disappeared but also was reversed: groups were now more cooperative than individuals. Data analysis on participants' strategies, survey questionnaires, and 
recorded discussions in Gong et al. (2009) revealed that three major factors (greed, fear, and persuasion power) that underlied the usual discontinuity effect were reduced in the stochastic environment.

Note that, in the SPD game, uncertainty not only reduced inter-group competitiveness but also encouraged inter-group cooperativeness. Although some possible explanations were proposed for the group cooperativeness under uncertainty in Gong et al. (2009), we could not verify or refute any of them as they were based on previous literature rather than empirical studies.

There are at least three reasons underlying an agent's decision to invest in a SPD game. First, an agent may decide to invest to reduce her own risk because she has a high degree of risk aversion. As shown in Table 1 and 2, a risk neutral agent will never invest independent of her counterpart's action. But a highly risk averse agent will invest even when she expects her counterpart not to invest if the agent's risk preference is such that "paying the investment cost of 45 " is worth more than "reducing the chance of losing 100 from $52 \%$ to $20 \%$."

The second reason for investing is that the agent trusts her counterpart to invest, and decides to cooperate so as to remove the risk. In this case the agent prefers to "pay the investment cost of 45 " in order to "reduce the chance of losing 100 from $40 \%$ to zero". If, however, the agent expects her counterpart not to invest, she will also not invest. She thinks that "paying the investment cost of 45 " is not worth "reducing the chance of losing 100 from $52 \%$ to $20 \%$."

The third reason why an agent cooperated in the SPD game is because she is concerned with the welfare of others which we term the niceness norm. In this case a person may decide to invest because paying the investment cost is worth a reduction in risk to both herself and her counterpart.

This paper reports on two studies designed to determine the statistic significance and relative importance of each of the above three explanations regarding why groups cooperated in the SPD game. More specifically we tested three group processes that may lead groups to cooperate more than individuals to reduce risks: a group being more concerned with risks, having higher cooperation expectation of their counterpart group, and being under greater social pressure to conform to the niceness norm.

The paper is structured as follows. Section 2 and 3 present each of the two studies respectively by providing a rationale for the study, the experimental design, the results, and a discussion of the findings. Study I examines whether groups and individuals have different risk preferences. Study II is a comprehensive survey that examines the rationale for cooperation by focusing on possible group-individual differences in cooperation expectation and social pressure to conform to various norms. Section 4 examines the strengths and weaknesses of current studies, and concludes by suggesting future research directions. 


\section{Study I: Group-individual Risk Preference Difference}

\subsection{Rationale for the Study}

Previous research has shown that people suffer from guilty feelings if they inflict harm on others (Baumeister et al., 1994). Charness and his coauthors found that people tried to live up to others' expectations to avoid guilt (Charness and Dufwenberg, 2006). In Charness and Jackson (2009), 90\% of participants whose decisions were affected by having a silent partner played a less risky strategy when choosing for themselves and their partner than when playing only for themselves so to avoid ex-post guilt. Group members may also choose to cooperate to reduce risks in an effort to avoid ex-post blame (Gong et al., 2009). If one group member suggests Not Invest and a large loss occurs later, then other group members may blame her for the loss.

In the SPD game, although cooperation (Investing) has a lower expected payoff than defection (Not Investing), cooperation reduces the risk of the agent suffering a larger loss and is considered a safer option than defection. Hence, both guilt aversion and blame avoidance encourage group members to choose cooperation, the safer strategy.

\subsection{Study Design}

The Game. In Study I, participants played the same SPD game shown in Table 1 and were aware that they were playing against a computerized agent that was programmed to invest with certain probabilities. The probabilities were known to the players before they made their decisions as to whether to Invest or Not Invest. This design adopted the basic structure and incentives of the SPD game, but removed any interactive motivations for cooperation, such as expectation of future cooperation or social pressure to be nice. The only benefit from an agent's investment was to reduce her own probability of suffering a loss. Investment by a human agent had no effect on the decision of the computer or on potential future benefits from mutual cooperation. The investment difference between groups and individuals in this game should thus be completely determined by their differences in risk preference.

Each player played multiple Supergames, each consisting of ten rounds. At the beginning of each Supergame, players were given 1500 Talers; one Taler was exchangeable for 2 cents in the individual condition, and 6 cents in the group condition divided equally between the three group members. Money accumulated from a previous Supergame did not carry over to the next one. The probabilities in each round that the computer was likely to invest were selected based on the average investment tendency of real players in Gong et al. (2009) and are shown in Table 3.

--Insert Table 3 here--

Hypothesis. The hypothesis of interest concerns the group-individual risk preference difference discussed in Section 2.1. We will refer it as the 'group risk concern hypothesis: 
H1 (group risk concern hypothesis): Groups are more risk concerned and invest more frequently to reduce risks than individuals do in an SPD game when both groups and individuals play against a computer with a known probability of investing.

Participants. 182 people participated in a between-subject study. 150 were in the Group condition (50 groups in total), and 32 were in the Individual condition. All were paid a $\$ 10$ show-up fee. About $20 \%$ of the participants were randomly chosen to be paid the dollar values of Talers they earned in Study I.

Procedure. The study was conducted in a behavioral lab of a northeastern university using Z-tree, a software for developing economic experiments (Fischbacher, 2007). There were two kinds of players: individuals and groups. Participants played the game either as a member of a 3-person group or an individual. In the group experiment the three group members made a collective decision and shared the final payoffs equally among themselves. Participants were instructed to make unanimous decisions or use a 2 to 1 majority rule.

Each individual or group used one computer to make its decisions. The computers were placed in two connecting rooms and in separate stations surrounded by cardboards to provide anonymity. Subjects were approximately 6 feet apart. All participants were aware that they would play against a computer whose investing probabilities were known to them before they made their own decisions.

Participants were told at the beginning of the experiment that their payoffs would be based on a show-up fee (\$10) and might also be based on their performance in the game. All participants were asked to complete a quiz that contained a variety of questions regarding the game, the procedure, decision method, payment information etc., before they started playing the game.

\subsection{Results}

When facing a computer player whose investing probability was known, groups invested $38 \%$ of the time, and individuals invested $29 \%$ of the time. A logit regression confirmed that groups invested more frequently than individuals $(\mathrm{z}=2.25, \mathrm{p}<0.05)$, supporting H1 that groups were more risk concerned than individuals in the SPD game ${ }^{1}$.

More details on the investment decisions of groups and individuals in each round are illustrated in Figure 1. Besides supporting H1, Study I also revealed a few other interesting perspectives in groups and individuals' decision on risk management when playing with a computerized agent.

First, although there was no interaction between the participants and the computer player, Figure 1 implies that participants responded to the investment probability changes of the computer over time as if there had been an interaction. The investment tendency of both groups and individuals decreased as the probability of

\footnotetext{
1 The group risk concern hypothesis was also confirmed in another independent study (Gong, 2009).
} 
computer investing decreased. One possible reason for this behavior is that participants might focus on the likelihood of investing so as to reduce the risk to zero, which is consistent with previous research on the desire of zero risk (Viscusi et al., 1987). When there was a higher probability that the computer would invest, it was more likely that the participants could completely remove the risk of losing 100 Talers by investing 45 Talers. When the computer's investment probability was low, however, the participants realized that investing 45 Talers by themselves would be unlikely to reduce the risk to zero.

--Insert Figure 1 here --

Second, although both groups and individuals responded to the probability changes, there are a few noticeable group-individual differences in terms of how they responded to the changes in the computer's decision to invest. As stated before, in general groups invested significantly more frequently than individuals, which supports H1. But the group-individual risk preference difference was not always of the same magnitude in all rounds with different investment probabilities. As shown in Figure 1, when the computer's probability of investing was high (Round 1 and 2), roughly the same percentage (around 40\%) of the groups and individuals thought that it was worth 45 Talers to have a high chance of removing the risk completely. As the probability of the computer investing and likelihood of removing the risk decreased (Round 3--8), groups and individuals illustrated different risk reduction tendencies. A higher percentage of groups than individuals chose to invest to reduce their own risk, even though they knew that the computer's probability of investing was low, which is also consistent with $\mathrm{H} 1$ that groups are more concerned about risks than individuals.

Third, the investment difference between groups and individuals in Study I (38\% vs. $29 \%$ ) was much smaller than that in Gong et al. (2009) (52\% vs. 22\%). The difference in the magnitude may imply that group risk reduction in the SPD game only partially explains the group cooperativeness. Another limitation of Study I is that, although it confirmed that groups are more risk-concerned than individuals, it did not provide specific information as to whether the group risk concern was driven by guilt aversion or blame avoidance or other unidentified factors.

\section{Study II: A Survey Study on the SPD game}

Study II was designed to investigate two other group processes that might have motivated groups to invest in the SPD game: the group cooperation-expectation hypothesis and the social norm hypothesis. We also examine underlying motivations for groups to become more risk concerned than individuals.

\subsection{Rationale and Hypotheses}

\subsubsection{The Group Cooperation-Expectation Hypothesis}

Besides the group risk concern explanation confirmed in Study 1, another reason why groups may have cooperated more than individuals in the SPD game is that groups 
may have higher expectations that their counterpart would invest than individuals after observing their own groups' high risk concern and cooperation tendency, and generalizing that to the other group.

H2 (group cooperation-expectation hypothesis): Groups have higher cooperation expectation for their counterpart groups than individuals have for their counterpart individuals under uncertainty.

Note that, the cooperation expectation argument (H2) is related to risk preference hypothesis $(\mathrm{H} 1)$, and explains group cooperation under uncertainty from a different perspective than the risk concern argument, risk concern is based on whether "acting as a group under uncertainty" pushes group members to be more focused on the likelihood and consequences of experiencing a loss, while the cooperation expectation argument considers whether "interacting with another group (rather than another individual) under uncertainty" changes players expectation as to how likely their counterpart will cooperate.

\subsubsection{The Social Norm Hypothesis}

The third potential explanation as to why groups cooperate more than individuals in the SPD game is that group members are under social pressure from other members to conform to certain social norms, especially the niceness norm which encourages cooperation.

When people are in a group and their behavior is observable to others, they are often under pressure to conform to social norms (see Turner, 1991 for a review on social conformity). In an interactive game such as the Prisoner's Dilemma, three norms are especially relevant: the norm of being pro-group (Tajfel, 1982), the norm of being smart (Orbell and Dawes, 1981), and the norm of being nice (Dawes 1980). For a more detailed discussion on these three norms in both DPD and SPD game, see Gong et al. (2009).

With uncertainty in the outcomes, there is no clear answer as to which strategy, Invest or Not Invest, is pro-group or smart. Defection has a higher expected payoff and may be regarded as a pro-group and smart strategy by some members. Other members may disagree because they are unwilling to take the risk associated with defection contending that cooperation reduces the risk and is thus a pro-group and smart strategy. The third norm, the niceness norm, clearly indicates that cooperation yields mutual benefits. We argue that it is possible that in the SPD game when defection cannot be easily justified by being pro-group and being smart, social pressure pushes group members to conform to the niceness and cooperation norm. This might be a third reason why groups may cooperate more than individuals to reduce risks. Thus, we test the following niceness norm hypothesis in Study II:

$\boldsymbol{H 3}$ (group niceness norm hypothesis): Group members are under greater social pressure (than individuals) to be nice and report "being nice" to be one reason why they choose to invest.

Besides the niceness norm hypothesis, we will also test whether group members 
are under greater social pressure than individuals to conform to a variety of other norms to shed more light on group decision process in the SPD game.

\subsubsection{Motivations underlying Group Risk Concern}

A third purpose of Study II is to investigate the underlying motivations that drive groups to be cautious about risks. We do this by asking group members to identify reasons why they do not suggest or defend defection strategy (Not Invest) even though they personally believe that defection is a good strategy. The study is thus designed to complement Study I which provided confirmation of the group risk concern hypothesis (H1) but did not provide specific information as to whether the group risk concern was driven by guilt aversion, blame avoidance, or other factors. Specifically we will test the following two hypotheses:

H1a: Group members identify ex-post guilt aversion as one of the reasons why they do not suggest or defend defection.

$\boldsymbol{H} 1 \boldsymbol{b}$ : Group members identify ex-post blame avoidance as one of the reasons why they do not suggest or defend defection.

In summary, we attempt to answer the following questions in Study II: 1) Do groups have higher cooperation expectation of their counterparts than individuals have? 2) Does group membership or group process put social pressure on group members to conform to certain norms? If yes, what are they? Are group members under social pressure from other members to conform to the niceness norm that encourages cooperation? 3) What factors drive the group-individual risk preference difference? Specifically, do ex-post guilt aversion and ex-post blame avoidance prevent group member from suggesting Not Invest and cooperate (Invest) more frequently to reduce the risk?

\subsection{Participants and Design}

There were three conditions in Study II, Individual Survey (IS), Group Private Survey (GPS), and Group Shared Survey (GSS). There were 32 participants in the IS condition, 72 participants (24 groups) in the GPS condition, and 78 participants (26 groups) in the GSS condition (total, 182).

In the IS condition, all participants first read the instructions for the SPD game in Table 1, finished the quiz, then completed a survey that contained a series of questions on their strategy choice, expectation of their counterpart, and social preferences. In the two group conditions (GPS and GSS), participants were assigned to 3-person groups, briefly met the other members, then were led to relatively private locations to read the instructions, finish the quiz, and complete the survey independently. The survey in the group condition included questions similar to the ones in the individual survey, plus a set of group-process-related questions.

In the GPS condition, the participants were told that, upon the completion of the 
survey, the experimenter would collect the survey sheets before they rejoined their groups to discuss the strategies and play the game. That is, their survey results would remain private and other members in their groups would not see their answers. In the GSS condition, the participants were told that they would bring their surveys and share them with their group members later when they rejoined the group to play the game. Note that all participants answered the survey questions before they actually participated in a group discussion or played the game.

The initial motivation to have both private and shared group surveys was to distinguish between "group membership alone" and "group membership plus peer pressure." However, even in the GPS condition, the social pressure to conform to certain norms was not completely removed because group members might foresee intragroup discussion even though their survey would remain private. Such anticipation might lead them to take the perspective of being part of a group. Possibly for that reason, we did not observe statistically significant differences between the GPS and GSS conditions. Hence the data from the two surveys were pooled for the analysis on group-individual differences.

\subsection{Results and Discussion}

To test the group cooperation-expectation hypothesis, we asked all players to estimate how likely they thought their counterpart would invest in the first round using a 100-point scale ranging from 0 (my counterpart will never invest) to 100 (my counterpart will always invest). Consistent with $\mathrm{H} 2$, groups showed a significantly higher expectation that their counterpart would invest than individuals did (64 vs. $54, t(180)=1.63, p=0.05$ one-tail t-test). Note that this difference could be magnified in later rounds because the first-round decision based on the first-round expectation set the players' initial tendency to cooperate and could have an impact on the general strategy the players adopted in later rounds of the game.

To investigate whether being in a group imposed extra social pressure to conform to certain social norms, participants were asked to rate two sets of norms on a 0 -100 scale where 0 represents "it does not describe me in any situation" and 100 represents "it describes me in all situations." The first set was four positive norms: desire to be regarded as being smart, being a team player, being nice, and working hard. The second set was four negative norms: desire not to be regarded as being selfish, being gullible, being incompetent, and being lazy.

We ran a logit regression using the player type (group or individual) as the dependent variable, and 8 social norm questions as independent variables, then performed an ANOVA based on the logit regression. No significant difference between group members and individuals were found $\left(\chi^{2}(8)=11.29, p>0.10\right)$, which indicated that at least before group discussion, social norms did not appear to play a role in the SPD game. This finding does not support $\mathrm{H} 3$ which proposes that the niceness norm plays a role in leading groups to cooperate. 
We examine $\mathrm{H} 1 \mathrm{a}$ and $\mathrm{H} 1 \mathrm{~b}$ in a set of questions about why group members hesitated to suggest or defend defection when other members made a cooperative suggetion in the SPD game. In other words, what factors drive groups to be cautious about risks and choose the cooperative and risk-reduction strategy (Invest) over the defective and expected-payoff dominant strategy (Not Invest)? Seventy-seven out of 150 group members indicated that they would not suggest or defend defection because they believed that defection was a bad strategy. What is more interesting is why the other 83 group members who believed that defection was a good strategy, would not promote the defective strategy. Those group members were asked to choose reasons for not suggesting or defending defection on a series of closed-ended ratings, followed by one open-ended question for any reasons that might have been missing in the closed-ending ratings. Several participants answered the open-ended question, but their answers were reflected closed-ended options and were transformed into closed-ending answers.

Table 4 shows the importance of each of the five reasons for participants not suggesting or defending defection. Note that the percentages sum to more than 100 percent because participants were asked to identify all applicable reasons for their behavior.

--Insert Table 4 here--

As shown in Table 4, guilt aversion (54\%) and blame avoidance (69\%), turned out to be two major motivations for not defecting, thus providing support for H1a and H1b, This finding is consistent with previous research (Baumeister et al. 1994; Charness and Jackson, 2009) and suggests why groups choose cooperation more frequently than individuals do. Again, there was little evidence supporting H3 that group members did not suggest defection (Not Invest) because of violating the niceness norm (4\%).

\section{General Discussion and Conclusion}

Previous research has shown that groups are usually less cooperative than individuals in prisoner's dilemma games. This well-replicated phenomenon is termed the "discontinuity effect". In a recent paper, we replicated the discontinuity effect that groups cooperated less than individuals in a deterministic Prisoner's Dilemma game, but found a reversed discontinuity effect that groups cooperated more than individuals in a stochastic version of the Prisoner's Dilemma, in which cooperation reduces risks (Gong et al., 2009).

The current paper extends the research on group cooperation under uncertainty by investigating the rationale underlying group behavior. Two studies examined the following possible reasons for group-individual cooperation differences under uncertainty: risk preference differences, cooperation expectation difference, and social pressure in groups to conform to social norms.

These two studies reveal that guilt aversion and blame avoidance drove group members to be more cautious and have higher risk concern than individuals. Hence it was 
more likely for groups to cooperate and invest so as to reduce their risk than individuals did. Groups also have a greater expectation of cooperation of their counterpart groups than individuals did of their counterparts. This is probably due to groups generalizing their own high risk aversion and cooperation tendency to their counterparts. The current studies show no evidence of social pressure pushing groups to be nicer and more cooperative than individuals.

The finding that guilt aversion and blame avoidance encourage groups to cooperate has important policy implications. To illustrate, imagine that two villages are located close to a lake and households in each village need to make a decision as to whether they are willing to pay a cost to get their waste disposed or directly dump their refuse into the lake, thus increasing the health risk for all households. According to the findings from Studies I and II, the two villages have a better chance of protecting the lake from being polluted if they each establishe a committee to make a collective decision rather than having each household make individual decisions. Similar reasoning can be applied to numerous scenarios in which there is an interdependent security problem and cooperation reduces each other's risks. Under those circumstances, groups have a better chance of cooperating and hence managing their risks more efficiently. This may also partly explains why people consistently trust groups over individuals on important decisions, in spite of the well-known fact in social psychology that groups are usually worse than individuals in terms of cooperation.

The group risk concern phenomenon is also of theoretical interest, because our finding conflicts with a well-known phenomenon in social psychology called group risk polarization or the risky shift, which states that there is a tendency for groups to behave in a more extreme manner than if individual members made choices on their own. Groups will become more risk averse only if individual members have a risk-averse tendency. If, however, individual members initially prefer risk-seeking, groups will be more risk seeking than individuals.

The group polarization prediction is inconsistent with behavior in Study I in which individuals invested $29 \%$ of the time to reduce risks and groups invested $38 \%$ of the time. Group polarization theory would have predicted that groups invest less than $29 \%$ of the time. There are at least two possible reasons for why group polarization fails to predict group risk concern in the current game. First, groups in the polarization studies are usually asked to report their risk preferences only, while in the current study groups can take an active role in reducing risks. Second, previous group polarization involves no interaction between groups, while the current study is an interactive and strategic game. Although a detailed discussion on the congruency and disparity between group risk concern and group risk polarization is beyond the scope of this paper, one of the authors is conducting a series of studies to further investigate the phenomenon.

One question that needs to investigated in future research is "when do groups cooperate more than individuals?", or the generality question. The game we have been using involves several unique features that may affect decisions regarding group cooperation so one needs to be cautious in generalizing to other situations. For example, 
the current study involved a game with negative outcomes in which players invest to reduce the risks of suffering a loss. It is unclear what would happen in an investment game with positive outcomes. Researchers have found that people encode losses and gains differently (Seymour et al., 2007) and typically show greater sensitivity to losses than to gains (Kahneman and Tversky, 1979). The guilt-aversion and blame-avoidance motivations underlying the reversed discontinuity effect may be stronger in a loss-domain game than in a gain-domain game because, if a loss occurred, the regret from not investing in risk-reducing measures is likely to be larger than if additional revenue could have been earned from investing in a profit-enhancing measure in a gain-domain game. It will be interesting to test whether there is a triple interaction between the loss/gain domain, the group/individual player type, and the DPD/SPD game type.

The nature of the uncertainty is another factor that may interact with group cooperation. As shown in Table 1, in the upper left cell, both players investing can completely remove the uncertainty. What if the risk is irremovable? For example, many environmental risks have two parts, uncertainty due to nature, and uncertainty due to human activity. Even if one stopped emitting greenhouse gases immediately, it is likely that global warming will still occur. Based on previous discussion, we would expect the level of guilt aversion and blame avoidance to be lower because there is no longer an outcome that has zero risk. Thus, we may expect to see lower risk-reduction driven cooperative behavior from groups.

There are other factors that may have an effect on group cooperation decisions, including, but not exclusive to, leadership of the groups, existence of competition with a group, group decision making (voting) rules, the length of the game, communication between players, the incentive structure of the game, the number of players, etc.

Group cooperation under uncertainty is a new phenomenon with many perspectives remaining to be explored. Better understanding the phenomenon will have broad implications for developing risk management strategies for important societal problems with interconnected networks for improving individual/group and social welfare. 
Table 1: Possible outcomes in the SPD game

\begin{tabular}{|c|c|c|c|}
\hline & \multicolumn{2}{|c|}{ Player 2} \\
\hline & & Invest & Not Invest \\
\hline \multirow{2}{*}{$\begin{array}{c}\text { Player } \\
1\end{array}$} & Invest & $-45 ;-45$ & $\begin{array}{l}20 \% \text { lose } 145,80 \% \text { lose } \\
45 ; \\
40 \% \text { lose } 100,60 \% \text { lose } \\
0\end{array}$ \\
\hline & Not Invest & $\begin{array}{l}40 \% \text { lose } 100,60 \% \\
\text { lose 0; } \\
20 \% \text { lose } 145,80 \% \\
\text { lose } 45\end{array}$ & $\begin{array}{l}52 \% \text { lose } 100,48 \% \text { lose } \\
0 ; \\
52 \% \text { lose } 100,48 \% \text { lose } \\
0\end{array}$ \\
\hline
\end{tabular}

Table 2: Possible outcomes in the DPD game

\begin{tabular}{|l|l|l|l|}
\hline \multicolumn{2}{|c|}{} & \multicolumn{2}{c|}{ Player 2 } \\
\cline { 3 - 4 } \multicolumn{2}{|c|}{ Player 1 1} & Invest & Not Invest \\
\cline { 3 - 4 } & Not Invest & $-45 ;-45$ & $-65 ;-40$ \\
\hline
\end{tabular}

Table 3: Invest Probabilities of the Computer Player in Study I

\begin{tabular}{|l|l|l|l|l|l|l|l|l|l|l|}
\hline Round Number & 1 & 2 & 3 & 4 & 5 & 6 & 7 & 8 & 9 & 10 \\
\hline $\begin{array}{l}\text { Probability of } \\
\text { Investing }\end{array}$ & $60 \%$ & $55 \%$ & $54 \%$ & $53 \%$ & $50 \%$ & $49 \%$ & $47 \%$ & $42 \%$ & $31 \%$ & $17 \%$ \\
\hline
\end{tabular}


Table 4: Reasons for Not Suggesting Defection in Study II

\begin{tabular}{|c|c|}
\hline Blame Avoidance & $69 \%$ \\
\hline Guilt Aversion & $54 \%$ \\
\hline Persuasion Concern & $30 \%$ \\
\hline Does Not Care Enough & $25 \%$ \\
\hline Niceness Norm & $4 \%$ \\
\hline
\end{tabular}


Figure 1: Group-Individual Investments when Playing with a Computer in Study I

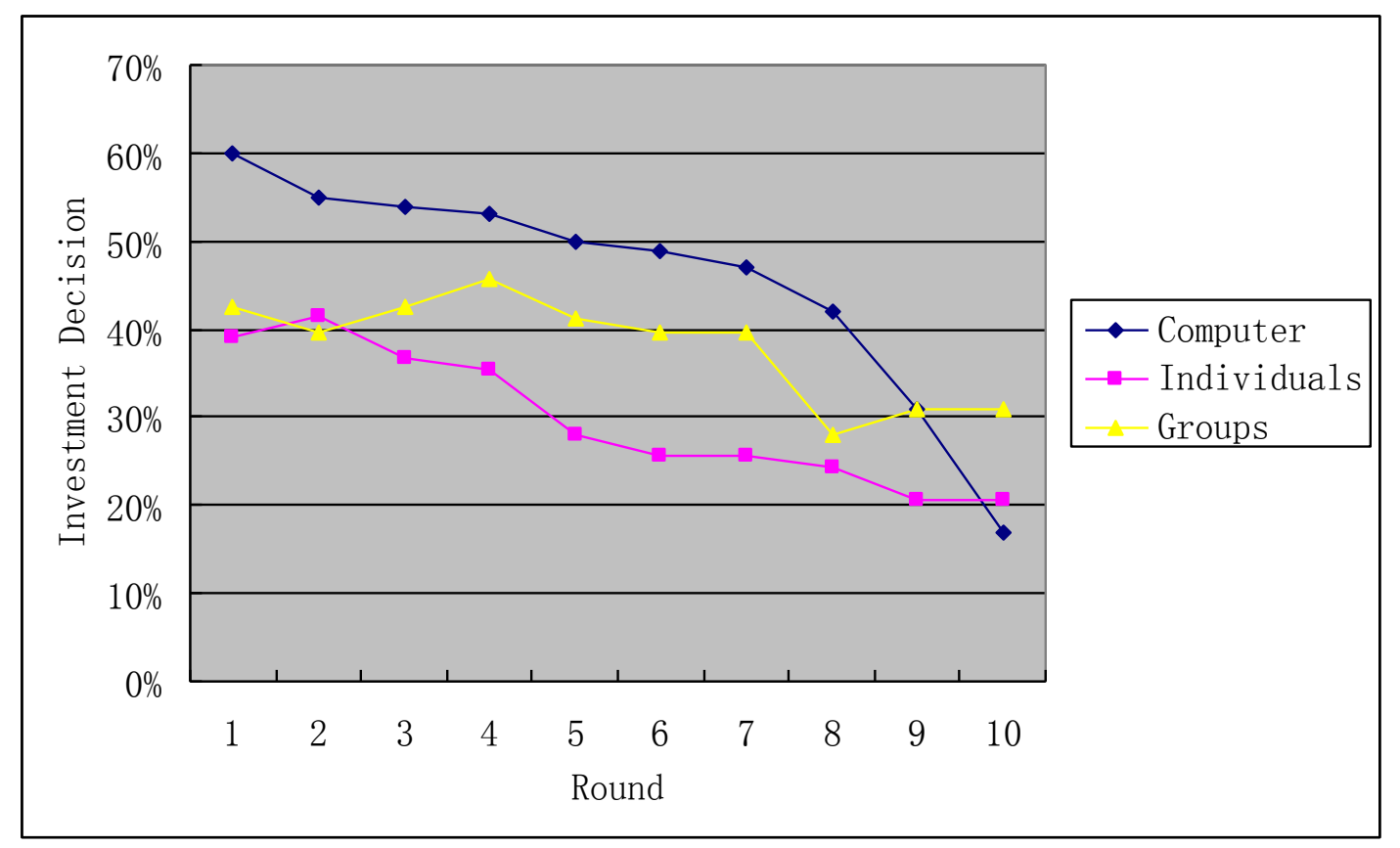




\section{References}

Baumeister, R. F., Stillwell, A. M., Heatherton, T. F. (1994). Guilt: An interpersonal approach. Psychological Bulletin, 115, 243-267.

Charness G., Dufwenberg, M. (2006). Promises and partnership. Econometrica, 74(6), 1579-1601.

Charness, G., Jackson, M.O. (2009). The role of responsibility in strategic risk-taking. The Journal of Economic Behavior and Organization , 69(3):241-247.

Dawes, R.M. (1980). Social dilemmas. Annual Review of Psychology, 31, 169-93.

Fischbacher, U. (2007). z-Tree: Zurich Toolbox for Ready-made Economic Experiments. Experimental Economics, 10(2), 171-178.

Gong, M., Baron, J. and H. Kunreuther. (2009). “Group Cooperation Under Uncertainty”. Journal of Risk and Uncertainty, 39(3): 251-270.

Gong, M. (2009b). “Group Cooperation under Uncertainty.” Ph.D dissertation, University of Pennsylvania, Philadelphia, PA.

Heal, G., Kunreuther, H. (2007). Modeling interdependent risks, Risk Analysis 27(3), 621-634.

Kahneman, D., Tversky, A. (1979). Prospect theory: an analysis of decisions under risk. Econometrica, 47, 313-327.

Kunreuther, H. (2009), “The Weakest Link: Managing Risk Through Interdependent Strategies" in Network Challenge: Strategy, Profit and Risk in an Interlinked World, P.R. Kleindorfer and Y. Wind (eds.), Wharton School Publishing, Upper Saddle River, NJ.

Seymour, B, Daw, N.D., Dayan, P., Singer, T., Dolan, R.J. (2007). Differential encoding of losses and gains in the human striatum. Journal of Neuroscience 27, 48264831 .

Tajfel, H. (1982). Social psychology of inter-group relations. Annual Review of Psychology, 33, 1-39. 
Turner, J.C. (1991). Social Influence. Brooks/Cole Publishing Company, Pacific Grove, California.

Wildschut, T., \& Insko, C. A. (2007). Explanations of interindividual-intergroup discontinuity: A review of the evidence. European Review of Social Psychology, 18, 175-211.

Viscusi, W. Kip, Wesley A. Magat \& Joel Huber (1987). An investigation of the rationality of consumer valuations of multiple health risks. RAND Journal of Economics, 18.4 (winter) 465-479. 